\title{
Methods Report \\ Cloning and Expression of Human Membrane-Bound and Soluble Engineered T Cell Receptors for Immunotherapy
}

\author{
Nehad M. Alajez, Saman Eghtesad, and Olivera J. Finn \\ Department of Immunology, School of Medicine, University of Pittsburgh, Pittsburgh, PA 15261, USA
}

Received 28 June 2005; Revised 15 November 2005; Accepted 21 November 2005

We report here the design and construction of several gene vectors for expression in mammalian cells of membrane-bound and soluble human T cell receptors (TR). We designed a vector (TR-ALPHA-IRES-TR-BETA pEF4) that encodes high-level expression of the full-length TR on the surface of T cells. Furthermore, we engineered TR that does not require the presence of endogenous CD3 molecules for surface expression and thus expression is not limited to T cells. We also constructed a vector encoding a single-chain TR (scTR) as a fusion protein of V-ALPHA-V-BETA-C-BETA with CD3Z. Since it is encoded and expressed as a single molecule, this scTR is well suited for gene therapy. Lastly, we successfully used a mammalian expression vector for generation of soluble human TR. The approaches we used here for manipulation of a human tumor-specific TR can be useful for other investigators interested in TR-based immunotherapy.

Copyright (c) 2006 Nehad M. Alajez et al. This is an open access article distributed under the Creative Commons Attribution License, which permits unrestricted use, distribution, and reproduction in any medium, provided the original work is properly cited.

\section{INTRODUCTION}

Failure of standard cancer therapies and their extreme toxicity have led to an increasing interest in utilizing the immune system to fight tumors. Adoptive transfer of in vitro expanded tumor-infiltrating lymphocytes (TILs) in conjunction with high dose IL-2 to advanced melanoma patients resulted in objective clinical responses [1]. Limitations for using adoptive transfer of tumor-reactive $\mathrm{T}$ cells range from the difficulty of obtaining sufficient numbers of TILs from most cancer patients to the limited in vivo survival of adoptively transferred $\mathrm{T}$ cells. To overcome these limitations several investigators have reported successful cloning of $\mathrm{T}$ cell receptor alpha (TR-ALPHA) and T cell receptor beta (TR-BETA) sequences from tumor-reactive $\mathrm{T}$ cell clones and transduction into in vitro activated primary $\mathrm{T}$ lymphocytes $[2,3]$.

Several reports in the literature have shown that functional interaction between a $\mathrm{T}$ cell and its target is dependent on the density and avidity of the TR for its ligand [4-8]. Therefore, for effective immune therapy involving TR gene transfer, it is necessary to design gene vectors that give the highest level of surface expression of the TR. Many solutions to specific problems involved in good TR expression from gene vectors can be found in the literature, but each applies to a different TR recognizing a different antigen. Thus, it has been difficult to directly compare specific solutions and to define some universally applicable experimental approaches. The purpose of our study was to design different constructs and expression vectors for one TR specific for the tumor antigen MUC1 for potential use in cancer immunotherapy, and to compare the level of cell surface TR expression achieved through different constructs on various cell types. In addition, we engineered and tested several constructs to obtain a soluble form of the TR that could be used for therapeutic purposes as well as to study the interaction between the TR and its ligand. These vectors could be very useful tools for investigators who are interested in studying TR function or TR-based immunotherapy.

\section{MATERIALS AND METHODS}

\section{Primers}

Sequences of all primers (P1-P15) used for cloning and the gene bank accession numbers for the correspondent genes are listed in Table 1. 
TABLE 1: Nucleotide sequences and restriction enzyme cleavage.

\begin{tabular}{|c|c|c|}
\hline Primer no & Accession no & Sequence \\
\hline P1 & DQ269212 & 5'-CGGGATCCTCGAGATGGAGACCCTCTTGGGCCTGCTTA-3' \\
\hline $\mathrm{P} 2$ & DQ269213 & 5'-CGGGATCCGTCGACATGGCCACCAGGCTCCTCTGCTG-3' \\
\hline P3 & DQ269212 & 5'-CGGGATCCGGAATTCTCAGCTGGACCACAGCCGCAGCGT-3' \\
\hline $\mathrm{P} 4$ & DQ269213 & 5'-ATAGTTTAGCGGCCGCGGATCCTCAGAAATCCTTTCTCTTGACCA-3' \\
\hline P5 & J04132 & 5'-GGGGATCCCAAACTCTGCTACCTGCTGG-3' \\
\hline P6 & J04132 & 5'TCCCCGCGGCGGCCGCGAATTCTTAGCGAGGGGGCAGGGCCTGCATG-3' \\
\hline P7 & DQ269212 & 5'-CGGGATCCAGATCCCCACAGGAACTTTCTGGGCTGGGGAAG-3' \\
\hline P8 & DQ269213 & 5'-CGGGATCCAGATCCCCACAGTCTGCTCTACCCCAGGCCTCG-3' \\
\hline P9 & DQ269213 & 5'-AGGCGCGCCCCCAGGCCTCGGCGCTGACGATC-3' \\
\hline P10 & BT019811 & 5'-AGGCGCGCCGACATGGCCCTGATTGTGCTGGGGGGC-3' \\
\hline P11 & ВT019811 & 5'-AGGCGCGCCGACGCTGGGGATATGGCCCTGATTGTGCTGGG-3' \\
\hline P12 & DQ269213 & $\begin{array}{l}\text { 5'CTAAGCGTAGTCTGGGACGTCGTATGGGTACAGATCCTCTT } \\
\text { CTGAGATGAGTTTTTGTTCTACAACGGTTAACCTGGTC-3' }\end{array}$ \\
\hline P13 & DQ269213 & $\begin{array}{c}\text { 5'CCTGCAGGTCAATGGTGATGGTGATGATGCTTGTCATCG } \\
\text { TCATCCTTGTAGTCAGCGTCTGCTCTACCCCAGG-3' }\end{array}$ \\
\hline $\mathrm{P} 14$ & DQ269212 & $\begin{array}{l}\text { 5’ATGTGGCTGCAGAGCCTGCTGCTCTTGGGCACTGTGGCC } \\
\text { TGCAGCATCTCTGCACCCCAGGAGGTGACGCAGATTC-3' }\end{array}$ \\
\hline P15 & DQ269212 & $\begin{array}{l}\text { 5'CCATGGAGACAGACACACTCCTGCTATGGGTACTGCTG } \\
\text { CTCTGGGTTCCAGGTTCCACTGGTGACGCGGCCCAGGAGGTGACGCAGATTC-3'3 }\end{array}$ \\
\hline
\end{tabular}

\section{Cloning of full-length TR-ALPHA and TR-BETA sequences}

MA CTL clone, the source of the TR, was described before [9]. RT-PCR was performed using GeneAmp RT-PCR kit (Applied Biosystems, Foster City, Calif, USA) and using either V-ALPHA (P1) or V-BETA (P2) leader sequence specific forward primers and C-ALPHA (P3) or C-BETA (P4) reverse primers. The TR-ALPHA was cloned into the Xho I and EcoR I sites in the multiple cloning site (MCS) A and the TR-BETA was cloned into the Sal I and Not I sites in MCS B in the pIRES vector (Clontech Laboratories, Palo Alto, Calif, USA). The TR-ALPHA-IRES-TR-BETA cassette was then subcloned into the pEF4 mammalian expression vector (Invitrogen, Figure 1(a)).

\section{Construction of a two-chain T cell receptor and a single-chain $T$ cell receptor vectors for expression on $T$ cells and non-T cells}

Human CD3Z chain was cloned using forward primer (P5) and reverse primer (P6). This cloning strategy maintained an endogenous BamH I site at nucleotide number 80 in the extracellular domain of human CD3Z. PCR product was cloned into the pCDNA3.1 TA vector (Invitrogen). The extracellular domains of the TR-ALPHA and TRBETA sequences were cloned using (P1) or (P2) forward primers and $(\mathrm{P} 7)$ or $(\mathrm{P} 8)$ reverse primers, respectively. The CD3Z/pCDNA3.1 vector was digested with BamH I restriction enzyme (New England BioLabs, Beverly, Mass, USA) and the TR-ALPHA or TR-BETA sequences were cloned inframe with the CD3Z chain at the BamH I site (Figures 2(a), 2(b)). The TR-ALPHA-Z and TR-BETA-Z were subcloned into the pIRES vector at the Xho I/EcoR I and the Sal I/Not I restriction sites, respectively. Finally the TR-ALPHA-Z-IRESTR-BETA-Z cassette was subcloned into the pLNCX2 (Clontech) expression vector using Xho I and Not I restriction sites (Figure 2(c)). The scTR was constructed by cloning the TR V-ALPHA and joining into the TR V-BETA-C-BETA (terminating at 2nd-CYS) using flexible linker (GGGGS) 3 . The TR V-ALPHA-V-BETA-C-BETA was then ligated in-frame to the murine CD3Z chain. A linker encoding a thrombin cleavage site (GDLVPRGSSRLD) was introduced between the TR CBETA and the CD3Z transmembrane region. The scTR was cloned into the pEF6 TA vector.

\section{Construction of SCTR-CD4TM-hZ mammalian expression vectors}

The scV-ALPHA-V-BETA-C-BETA was amplified using VALPHA forward (P1) and C-BETA reverse (P9) primers and cloned into the pEF6 TA vector. An Asc I site was introduced at the $\mathrm{C}$-terminus in the $\mathrm{C}$-BETA region. The human CD4 transmembrane (TM) domain fused to the human CD3Z cytoplasmic domain was amplified from the hCD4Z vector (kindly provided by Dr Margo R. Roberts, University of Virginia, Va, USA) using forward (P10) and reverse (P6) primers. Asc I and Sac II sites were introduced into the forward and reverse primers, respectively, that allowed inframe ligation to the scTR extracellular domain. A modified 


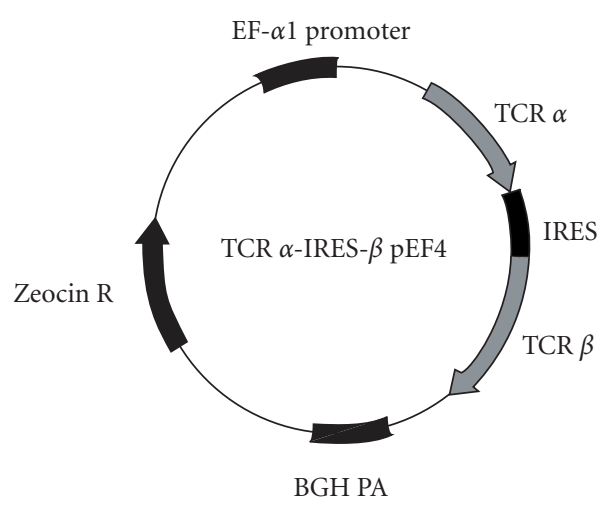

(a)

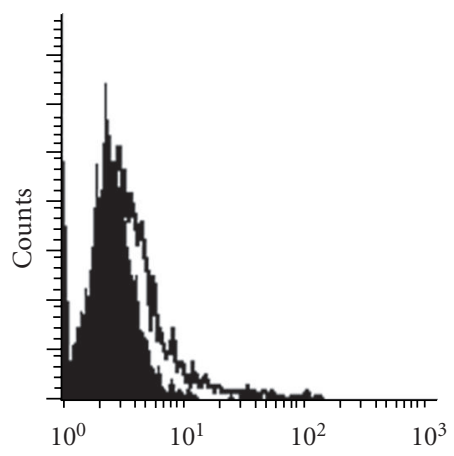

(c)

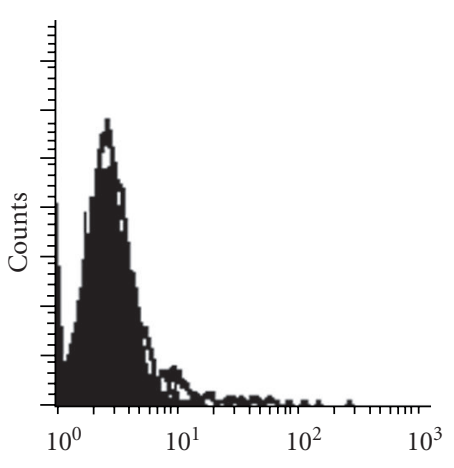

(b)

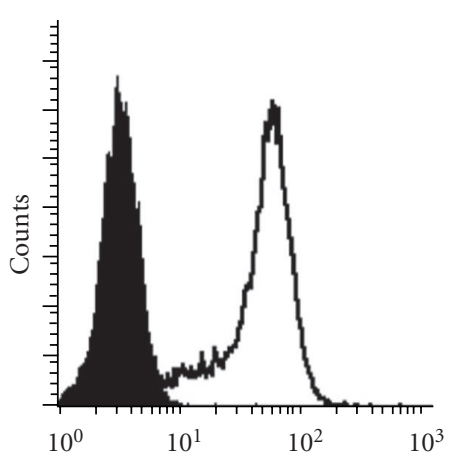

(d)

FIGURE 1: Expression of the TRAZ from MA CTL clone on the surface of a TR-deficient Jurkat line (JRT3-T3.5). (a) The TR-ALPHA-IRESTR-BETA cassette was cloned into the pEF4 expression vector. (b) Untransfected JRT3-T3.5, (c) JRT3-T3.5 cells transfected with the TCRB sequence, or (d) JRT3-T3.5 cells transfected with MA TR-ALPHA-IRES-TR-BETA pEF4 were stained with anti-CD3 Epsilon (open histogram) or with isotype control (filled histogram). IRES stands for internal ribosomal entry site. Zeocin is antibiotic resistance gene.

version of this vector was created by inserting three amino acid (AGD) linker between the C-BETA region and the CD4 TM domain. In the latter, PCR was done using (P6) and (P11) primers.

\section{Surface biotin labeling and thrombin cleavage of ScTR}

Surface biotin labeling and thrombin cleavage was done as previously described [10].

\section{Construction of secreted scTRs}

A soluble, single-chain fragment variable ( $\mathrm{scFv}$ ) encoding the TR V-ALPHA-V-BETA or soluble single-chain TR (soluble scTR) domain consisting of the TR V-ALPHA-V-BETA-CBETA (terminated at 2nd-CYS) was cloned into pCDNA3.1 TA vector by RT-PCR using (P1) and (P12) or (P2) and (P13) primers, respectively. Modified versions of the soluble scTR vector were created by fusing the sscTR to the GM-CSF, PCR was done using P14 and P13, or Ig- $\kappa$ light chain leader sequences (PCR was done using P15 and P13).

\section{Cell transfection}

Human embryonic kidney cells, HEK 293H, were transfected using lipofectamine 2000 (Invitrogen) according to manufacture's instruction. Cells were analyzed for protein expression 48-72 hours post-transfection. Jurkat cells were electroporated using a BioRad Gene Pulser II (Bio-Rad Laboratories, Hercules, Calif, USA) at $960 \mu \mathrm{F}$ and $200 \mathrm{~V}$ settings.

\section{Stimulation with superantigen}

BWZ and BWZ-scTR cells were stimulated with Staphylococcus enterotoxin E (SEE) (Toxin Technology, Sarasota, Fla) as described before [11]. Thirty-six hours later, IL-2 in culture supernatant was measured using murine IL-2 ELISA kit (BD PharMingen, San Diego, Calif) according to manufacture's recommendations.

\section{Purification of soluble scTR and western blotting}

Transfected 293H cells were grown in DMEM-10. Seventytwo hours after replacing with fresh medium, culture medium was harvested and used for protein purification. 


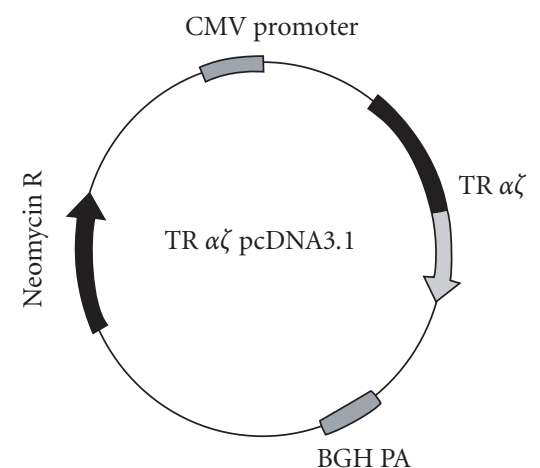

(a)

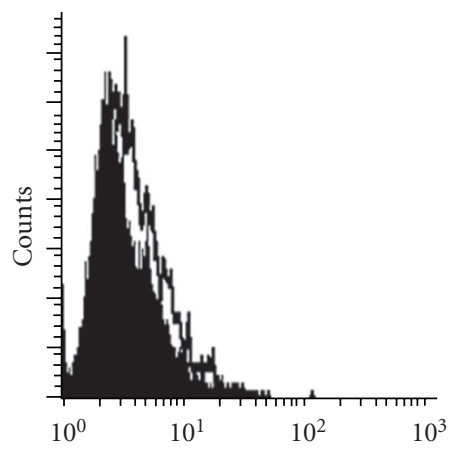

(d)

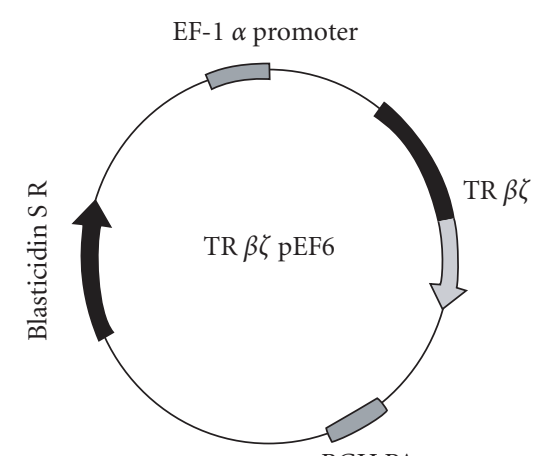

(b)

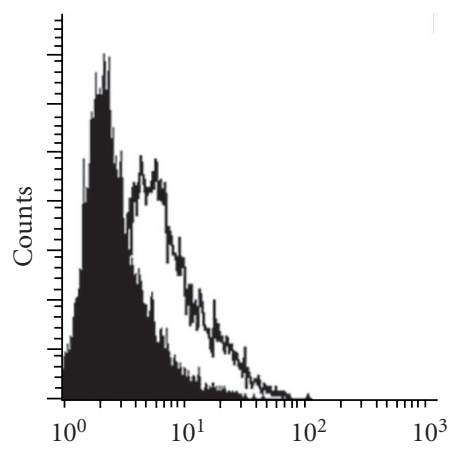

(e)

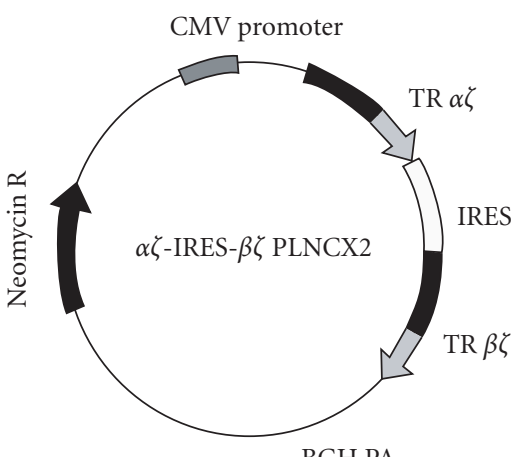

(c)

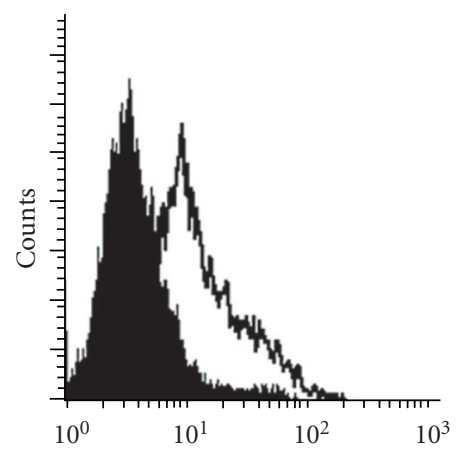

(f)

FIGURE 2: Construction and expression of engineered MUC1-specific TRAZ and TRBZ receptors. Expression vectors for (a) TRAZ, (b) BZ, and (c) AZ/BZ. (d) Untransfected $293 \mathrm{H}$ cells, (e) $293 \mathrm{H}$ cells cotransfected with the TRAZ and TRBZ, or (f) cells transfected with the AZ-IRESBZ pLNCX2 were stained for surface expression of the TR using anti-TR antibody BF1 (open histogram) or isotype control antibody (filled histogram).

Anti-HA, c-Myc, and 6-His antibodies were purchased from Santa Cruz Biotechnology, Santa Cruz, Calif, USA. AntiFlag M2 antibody was purchased from Sigma. For constructs encoding c-Myc or HA tagged proteins, soluble scTR was purified using protein G Sepharose beads (Amersham Biosciences) coated with the appropriate anti-tag antibody. For vectors encoding 6-His tagged proteins, soluble scTR was purified using nickel-agarose column (Qiagen) according to manufacture's recommendations.

\section{RESULTS}

\section{Reconstitution of the TR/CD3 complex on the surface of J.RT3-T3.5 Jurkat line transfected with the TR-ALPHA and TR-BETA construct}

J.RT3-T3.5 cells lack the TR-BETA transcript and have low levels of the TR-ALPHA chain message, and are thus suitable host cells for testing expression of transfected TRs. The pEF4 mammalian expression vector was chosen because expression of cloned genes is driven by the human elongation factor-1 alpha (EF-1 $\alpha)$ promoter, which is expected to be more transcriptionally active and stable in T cells than viral promoters. In addition, the presence of the IRES sequence permits expression of the TR-ALPHA and TR-BETA genes from the same message. This is expected to result in similar levels of expression of both genes, in contrast to genes driven by different promoters. As shown in Figure 1, untransfected JRT3-T3.5 did not express the TR/CD3 complex on their surface (Figure 1(a)). Transfection of the TR-BETA construct alone did not reconstitute the TR/CD3 complex on cell surface (Figure 1(b)); however, stable transfection of the TRALPHA-IRES-TR-BETA pEF4 vector into J.RT3-T3.5 cells resulted in high levels of TR/CD3 complex surface expression (Figure 1(c)). Cells transfected with the TR-ALPHA-IRESTR-BETA pEF4 vector recognized MUC1+ tumors in vitro \{Alajez, 2005 [12]\}.

\section{Engineered MUC1-specific two-chain TRs allow expression on the surface of non-T cells}

The requirement for CD3 molecules for expression of the $\mathrm{TR}$ on the cell surface limits its expression to $\mathrm{T}$ cells only. We constructed engineered vectors that would bypass this requirement and allow expression of TR on other cell types [10]. In $\mathrm{T}$ cells, the engineered vector eliminates any possibility that the transferred TRs could pair with endogenous TR-ALPHA or TR-BETA chains 
that would reduce the level of the specific receptor on the surface as well as form a potentially autoreactive TR [13]. Construction of the TR-ALPHA-Z and TR-BETA$Z$ was done as detailed in the "materials and methods." As shown in Figure 2, cotransfection of the TR-ALPHA-Z Figure 2(a) and TR-BETA-Z Figure 2(b) constructs into human epithelial kidney HEK $293 \mathrm{H}$ (non-T cell) cells resulted in surface expression of the TR-ALPHA-Z/TR-BETA-Z heterodimer (Figures 2(d) and 2(e)). Similar level of surface expression was also obtained when $293 \mathrm{H}$ cells were transfected with the TR-ALPHA-Z-IRES-TR-BETA-Z pLNCX2 vector (Figures 2(c) and 2(f)).

\section{Different configurations of single-chain TRs were expressed at various levels on the surface of $293 \mathrm{H}$ cells}

For clinical application, transfection with multiple plasmids is a cumbersome procedure. Other groups had previously reported successful construction and expression of single-chain TR (scTR) and single-chain antibodies [14, 15]. We made three different MUC1 scTR constructs to compare their efficiency by the levels of surface TR expression (Figure 3), scTR (shown in Figure 3(b)), scTR-CD4TMhZ (shown in Figure 3(c)), and the scTR-AGD-CD4TM-hZ (shown in Figure 3(d)). These scTRs consist of the TR antigen binding domain and a signaling component from the CD3Z chain. As shown in Figure 3(b), transfection of the scTR into $293 \mathrm{H}$ cells resulted in a substantial increase in surface expression compared to control (Figure 3(a)) but transfection with the scTR-CD4TM-hZ construct gave a much lower level of expression (Figure 3(c)). However, inserting a 3-amino-acid (AGD) linker between the TR C-BETA and the CD4 TM region restored high level of surface expression of the scTR (Figure $3(\mathrm{~d})$ ). It is possible that these 3 amino acids provided enough flexibility to the scTR to allow proper folding of the molecule and normal level of surface expression. Figure 3(e) shows the same results in a more quantitative way. High surface expression of the scTR was detected on both T cells (BWZ murine thymoma) and non-T cells (rat basophilic leukemia (RBL)) transfected with scTRpEF6 vector (Figure 4(a)). The scTR is functional as shown by the ability of the transfected BWZ cells to be stimulated with plate-bound anti-TR BF1 antibody or with SEE superantigen, which binds specifically to the human TRB8 region (Figure 4(b)). These cells also recognized MUC1+ tumor cells in vitro $\{$ Alajez, $2005[12]\}$.

\section{Multiple expression vectors for soluble scTR production}

Soluble (s) single-chain (sc) receptor (soluble scTR) could be used as vehicles to deliver and target therapeutic drugs to the site of their specific antigen (in our case a tumor expressing MUC1), or it can be used to study the affinity of interaction between the TR and its ligand using Biacore analysis. One approach we used to generate soluble scTR was to introduce a thrombin cleavage site between the
scTR C-BETA domain and the CD3Z transmembrane region (Figure 5(a)). Figure 5(b) shows that the scTR containing the thrombin cleavage site can be cleaved from the surface of transfected cells. Following thrombin cleavage, the scTR could be purified from the soluble fraction (Figure 5(c), lane 4) using an affinity column. As expected, the soluble scTR has a lower molecular weight than the membrane-bound scTR (Figure 5(c), lanes 2 and 4). The soluble scTR can be eluted from the affinity column under high $\mathrm{pH}$ elution conditions (Figure 5(c), lane 8). Even though this approach was successful in generating soluble scTR, the amount that was obtained was extremely low. Other groups have reported expression of a soluble, single-chain fragment variable $(\mathrm{scFv})$ domain of both antibody and $\mathrm{T}$ cell receptor [16, 17]. Figure 6(a) shows the design of the soluble scFv construct that encodes the TR V-ALPHA-V-BETA region. Figure 6(b) shows the soluble scTR construct that was terminated just before the 2nd-CYS in the TR C-BETA region. Two other constructs were designed as described in Figure 6(b), with the exception of replacing the V-ALPHA leader sequence in the soluble scTR with either a GM-CSF (Figure 6(c)) or Ig- $\kappa$ light chain (Figure 6(d)) signal peptide. Various epitope tags were inserted at the $\mathrm{C}$ terminus to facilitate protein expression and purification. When the soluble scFv construct was transfected into $293 \mathrm{H}$ cells, no recombinant soluble scFv protein could be detected in the culture supernatant (Figure 6(e), lane $\left.\mathrm{a}^{\prime}\right)$. However, transfection of the soluble scTR construct into $293 \mathrm{H}$ cells resulted in significant amounts of recombinant protein secreted in culture supernatants (Figure 6(e), lane $\left.b^{\prime}\right)$. Transfection of the soluble scTR that was fused to the Ig- $\kappa$ light chain leader sequence gave a lower level of protein expression than was seen in $b^{\prime}$ (Figure 6(e), lane $\left.\mathrm{d}^{\prime}\right)$, and transfection of the soluble scTR construct fused to the GM-CSF leader sequence yielded no protein secretion (Figure 6(e), lane $c^{\prime}$ ). These results showed that the presence of the TR C-BETA region is absolutely required for expression of the soluble scTR. We hypothesized that the TR CBETA region must be important for the proper folding of the protein or it interacts with and masks other hydrophobic amino acid residues in the TR V-BETA region, otherwise the $\mathrm{scFv}$ is rendered insoluble. As shown in Figure 6(f), the recombinant soluble scTR could be purified from culture supernatant using a nickel column. Figure 6(g) shows western blot analysis of purified fractions obtained in Figure 6(f), using anti-Flag M2 antibody. The expression o soluble form scTR in these mammalian cells appears to be sufficiently robust to produce this reagent for therapeutic purposes or for biophysical analyses.

\section{DISCUSSION}

In this report, we adapted several mammalian expression vectors for functional high-level expression of human TRA and TRB chains that can be useful for biological or biochemical analysis as well as immunotherapy. Our TRA-IRES-TRB pEF4 vector encoding the tumor antigen-specific TR generated high levels and stable expression of the TRA/TRB/CD3 complex on the surface of transfected $\mathrm{T}$ cells. We also 


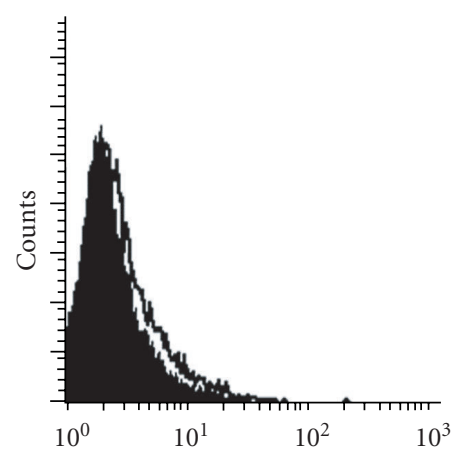

(a)

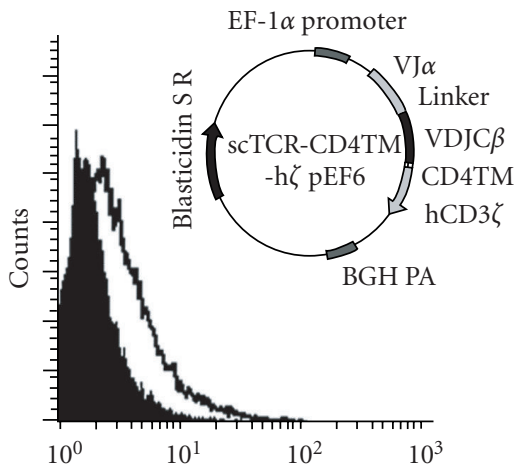

(c)

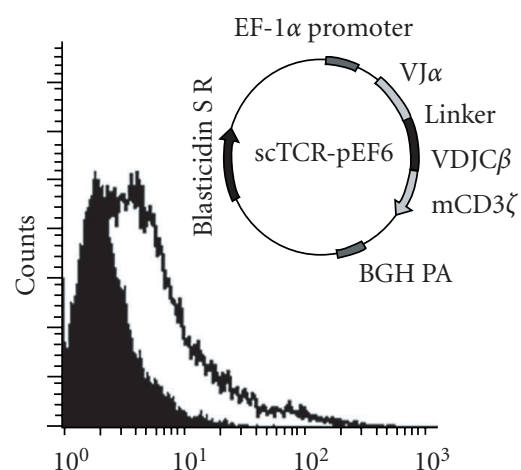

(b)

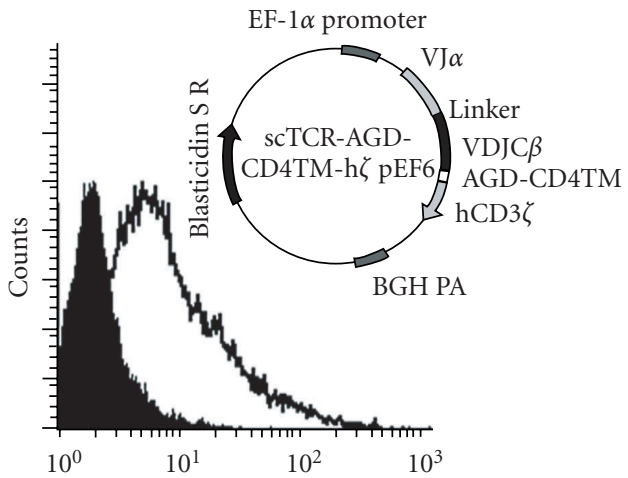

(d)

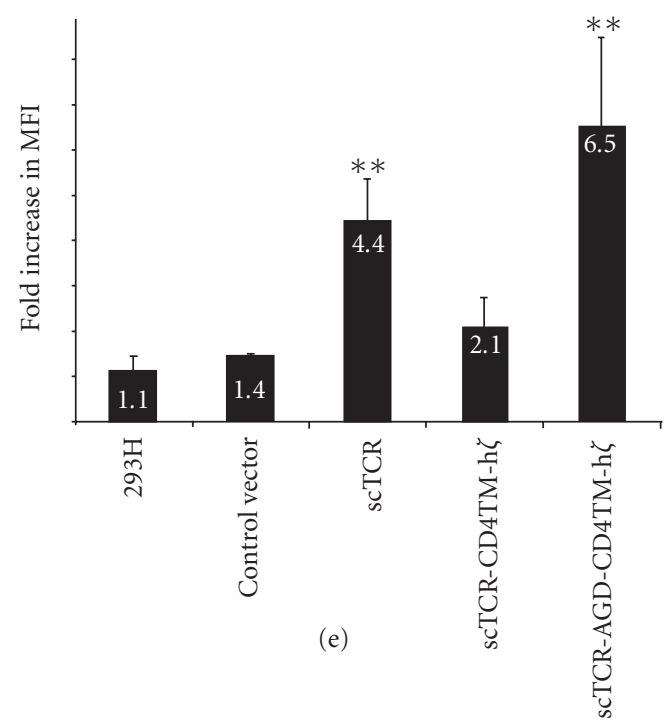

FIGURE 3: Construction and expression of MUC1-specific single-chain T cell receptors (scTRs). (a) 293H cells were transfected with (b) the scTR, (c) scTR-CD4TM-hZ, or (d) scTR-CD4TM-AGD-hZ mammalian expression vectors. Cells were stained with anti-TR BF1 (open histogram) or isotype control (filled histogram) antibody. (e) shows quantitative comparison of TR expression on $293 \mathrm{H}$ cells transfected with different scTR constructs. $P<.05$.

engineered TRAZ and TRBZ that were successfully expressed on the surface of $293 \mathrm{H}$ cells (a non- $\mathrm{T}$ cell line that does not express the CD3 complex). Additionally, we showed that surface expression of the TR was dependent on the coexpression of the TRAZ and TRBZ (data not shown). We hypothesized that pairing of the TRAZ to the TRBZ was crucial for proper folding and transport of the heterodimer through the endoplasmic reticulum (ER) and Golgi and eventually to the cell surface.

In contrast to the expression of two-chain TRs (tcTRs), functional scTRs can be expressed on the cell surface from a single mRNA transcript. In the example shown here we 


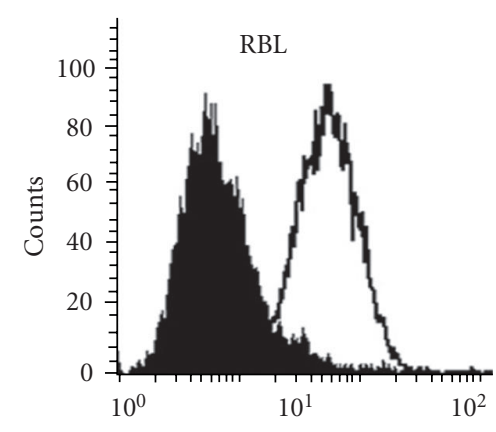

(a)

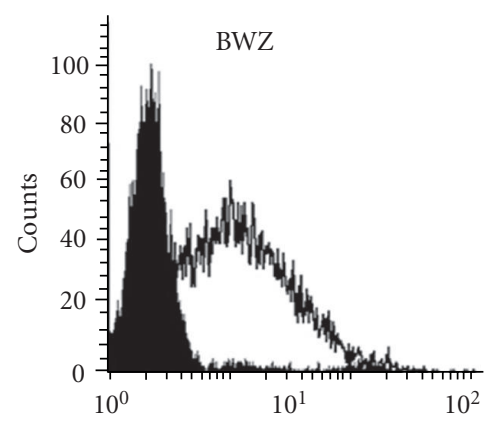

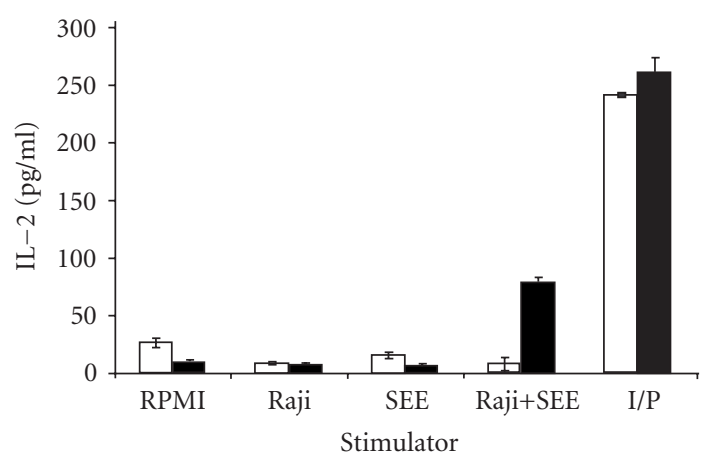

(b)

Figure 4: Expression of functional scTR on the surface of $T$ and non-T immune cells. (a) Rat basophilic leukemia (RBL) or BWZ cells were transfected with the scTR-pEF6 and were stained for surface expression with anti-TR BF1 antibody (open) or with isotype control (filled) histogram. (b) IL-2 secretion from BWZ cells (open bars) or BWZ-scTR (filled bars) following stimulated with SEE superantigen or with anti-TR BF1 antibody. Stimulation with Ionomycin/PMA (I/P) served as the positive control.

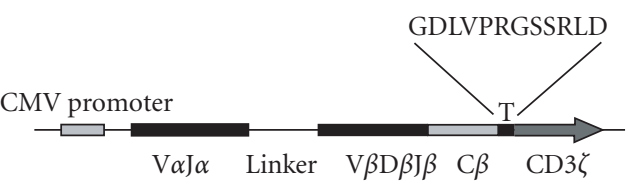

(a)
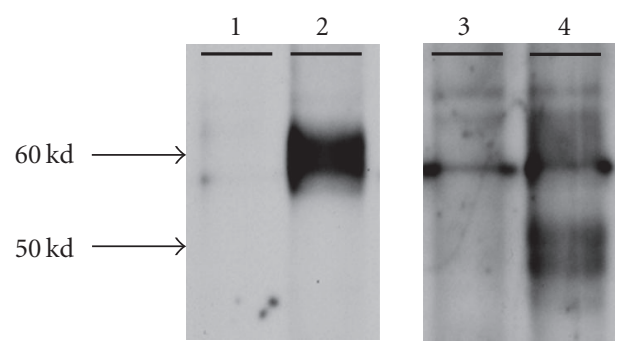

(c)

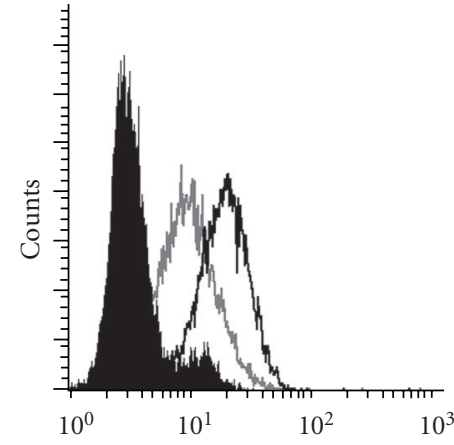

(b)

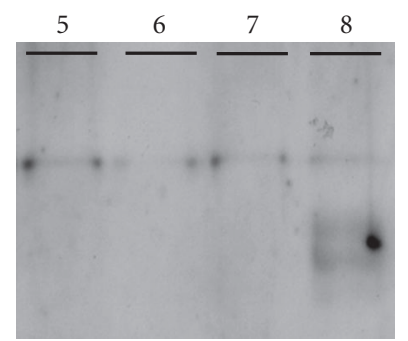

FIGURE 5: Expression and purification of soluble scTR following surface biotin labeling and immunoprecipitation. (a) scTR expression vector encoding a thrombin cleavage site, T. (b) RBL cells transfected with the scTR were stained with anti-TR BF1 antibody (open histograms) before (right) or after (left) treatment with thrombin. Filled histogram shows staining with isotype control antibody. (c) Immunoprecipitation of the scTR from RBL (lanes 1 and 3) or RBL cells transfected with the scTR (lanes 2 and 4) before (lanes 1 and 2) or after (lanes 3 and 4) treatment with thrombin. Lanes 6-8 are SA-HRP blotting of fraction eluted with $150 \mathrm{mM}$ Glycine, PH 2.2, $100 \mathrm{mM}$ Glycine PH 2.2, or diethylamine (DEA) PH 11.2, respectively. Lane 5 is IP from control lysate.

constructed a single-chain TR specific for the tumor antigen MUC1 and expressed it on the surface of different cell types. The expression of the scTR on the surface of transfected cells was lower than the level of expression of the native TR, which could be attributed to the presence of charged amino acids in the transmembrane (TM) domain of the CD3Z chain that might cause dimerization and retention of the scTR in the endoplasmic reticulum. In an attempt to increase the level of surface expression of the scTR, we replaced the TM domain of CD3Z in the scTR with the TM domain of human CD4. 


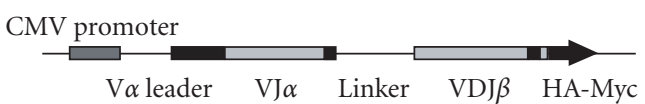

(a)

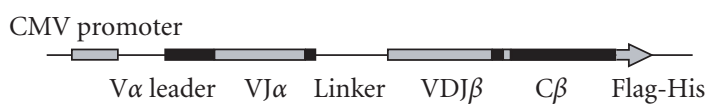

(b)

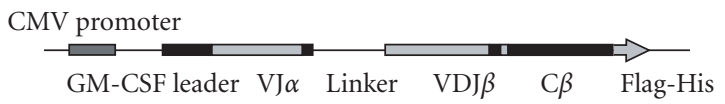

(c)

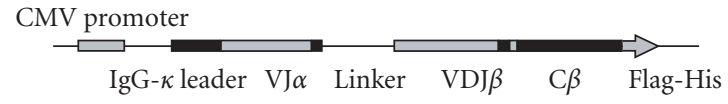

(d)

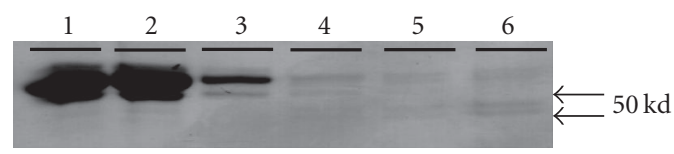

(f)

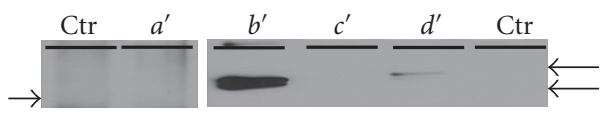

(e)

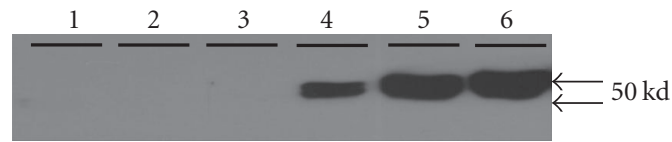

(g)

FIGURE 6: Expression and purification of soluble scTR using mammalian expression system. (a) The single-chain fraction variable (scFv) domain was cloned and fused to a C-terminus HA and c-myc epitope tags. (b) Secreted scTCR that was cloned and fused to a C-terminus Flag and 6-His epitope tags. (c) and (d) the secreted scTR as described in (b), with the exception that the secreted scTR was fused to the leader sequence from GM-CSF (c) or from Ig- $\kappa$ light chain (d). (e) Western blot of the culture supernatants from $293 \mathrm{H}$ cells transiently transfected with constructs $a-d\left(a^{\prime}-d^{\prime}\right)$, immunoprecipitated with appropriate anti-tag antibody and blotted with anti-c-myc antibody $\left(a^{\prime}\right)$ or with antiFlag-M2 antibody $\left(b^{\prime}-d^{\prime}\right)$. Ctr is supernatant from untransfected cells. (f) Coomassie blue staining of fractions from culture supernatant $\mathrm{b}^{\prime}$ purified using nickel column. Lane 1 is culture supernatant before purification, 2 is flow through, 3 is wash, and $4-6$ are different eluted fractions. (g) Western blot of panel (f) using anti-Flag M2 antibody.

However, this new construct was expressed at very low level until we inserted a 3-amino-acid (AGD) linker between the scTR constant region and the CD4 TM domain. High level of surface expression of the scTR was restored in this new construct. The short linker provided either flexibility or sufficient spacing between the TR constant region and CD4 TM to allow normal surface expression.

Most previous attempts to generate soluble TR were made using prokaryotic expression systems. However, proteins expressed in prokaryotic cells lack post-translational modifications and may be improperly folded. In order to avoid these potential problems, we chose to express soluble MUC1-specific scTR using mammalian expression systems. We terminated the scTR construct just before the last cystine in the TR C-BETA region. Following transfection into $293 \mathrm{H}$ cells, large amount of soluble scTR was detected in culture supernatants.

In conclusion the various constructs we adapted and tested for the expression of the MUC1-specific TR can be of interest and help to other investigators interested in TR immunotherapy or in studying TR-antigen interactions.

\section{ACKNOWLEDGMENTS}

This work was supported by the NIH Grant CA56103 to O. J. Finn and the DOD predoctoral training Grant DAMD1799-1-9352 to N. M. Alajez. We gratefully acknowledge the contribution of Dr Mark Alter and Dr Jan Schmielau to the early stages of this work.

\section{REFERENCES}

[1] Dudley ME, Wunderlich JR, Robbins PF, et al. Cancer regression and autoimmunity in patients after clonal repopulation with antitumor lymphocytes. Science. 2002;298(5594):850854.

[2] Rubinstein MP, Kadima AN, Salem ML, et al. Transfer of TCR genes into mature $T$ cells is accompanied by the maintenance of parental T cell avidity. The Journal of Immunology. 2003;170(3):1209-1217.

[3] Morgan RA, Dudley ME, Yu YY, et al. High efficiency TCR gene transfer into primary human lymphocytes affords avid recognition of melanoma tumor antigen glycoprotein 100 and does not alter the recognition of autologous melanoma antigens. The Journal of Immunology. 2003;171(6):3287-3295.

[4] Aarnoudse CA, Krüse M, Konopitzky R, Brouwenstijn N, Schrier PI. TCR reconstitution in Jurkat reporter cells facilitates the identification of novel tumor antigens by cDNA expression cloning. International Journal of Cancer. 2002; 99(1):7-13.

[5] Derby MA, Wang J, Margulies DH, Berzofsky JA. Two intermediate-avidity cytotoxic $\mathrm{T}$ lymphocyte clones with a disparity between functional avidity and MHC tetramer staining. International Immunology. 2001;13(6):817-824.

[6] Snyder JT, Alexander-Miller MA, Berzofsky JA, Belyakov IM. Molecular mechanisms and biological significance of CTL avidity. Current HIV Research. 2003;1(3):287-294. 
[7] Weijtens ME, Hart EH, Bolhuis RL. Functional balance between $\mathrm{T}$ cell chimeric receptor density and tumor associated antigen density: CTL mediated cytolysis and lymphokine production. Gene Therapy. 2000;7(1):35-42.

[8] Yang S, Linette GP, Longerich S, Haluska FG. Antimelanoma activity of CTL generated from peripheral blood mononuclear cells after stimulation with autologous dendritic cells pulsed with melanoma gp100 peptide G209-2M is correlated to TCR avidity. The Journal of Immunology. 2002;169(1):531-539.

[9] Magarian-Blander J, Ciborowski P, Hsia S, Watkins SC, Finn OJ. Intercellular and intracellular events following the MHCunrestricted TCR recognition of a tumor-specific peptide epitope on the epithelial antigen MUC1. The Journal of Immunology. 1998;160(7):3111-3120.

[10] Engel I, Ottenhoff TH, Klausner RD. High-efficiency expression and solubilization of functional $\mathrm{T}$ cell antigen receptor heterodimers. Science. 1992;256(5061):1318-1321.

[11] Mittelbrunn M, Yáñez-Mó M, Sancho D, Ursa A, SánchezMadrid F. Cutting edge: dynamic redistribution of tetraspanin CD81 at the central zone of the immune synapse in both T lymphocytes and APC. The Journal of Immunology. 2002; 169(12):6691-6695.

[12] Alajez NM, Schmielau J, Alter MD, Cascio M, Finn OJ. Therapeutic potential of a tumor-specific, MHC-unrestricted T-cell receptor expressed on effector cells of the innate and the adaptive immune system through bone marrow transduction and immune reconstitution. Blood. 2005;105(12):4583-4589.

[13] Willemsen RA, Weijtens ME, Ronteltap C, et al. Grafting primary human $\mathrm{T}$ lymphocytes with cancer-specific chimeric single chain and two chain TCR. Gene Therapy. 2000;7(16):13691377.

[14] Novotny J, Ganju RK, Smiley ST, et al. A soluble, single-chain T-cell receptor fragment endowed with antigen-combining properties. Proceedings of the National Academy of Sciences of the United States of America. 1991;88(19):8646-8650.

[15] Eshhar Z, Bach N, Fitzer-Attas CJ, et al. The T-body approach: potential for cancer immunotherapy. Springer Seminars in Immunopathology. 1996;18(2):199-209.

[16] Grégoire C, Lin SY, Mazza G, Rebai N, Luescher IF, Malissen B. Covalent assembly of a soluble T cell receptor-peptidemajor histocompatibility class I complex. Proceedings of the National Academy of Sciences of the United States of America. 1996;93(14):7184-7189.

[17] Pavlinkova G, Colcher D, Booth BJ, Goel A, Batra SK. Pharmacokinetics and biodistribution of a light-chain-shuffled CC49 single-chain Fv antibody construct. Cancer Immunology, Immunotherapy. 2000;49(4-5):267-275. 


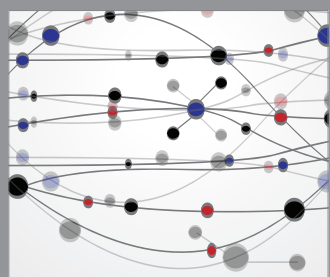

The Scientific World Journal
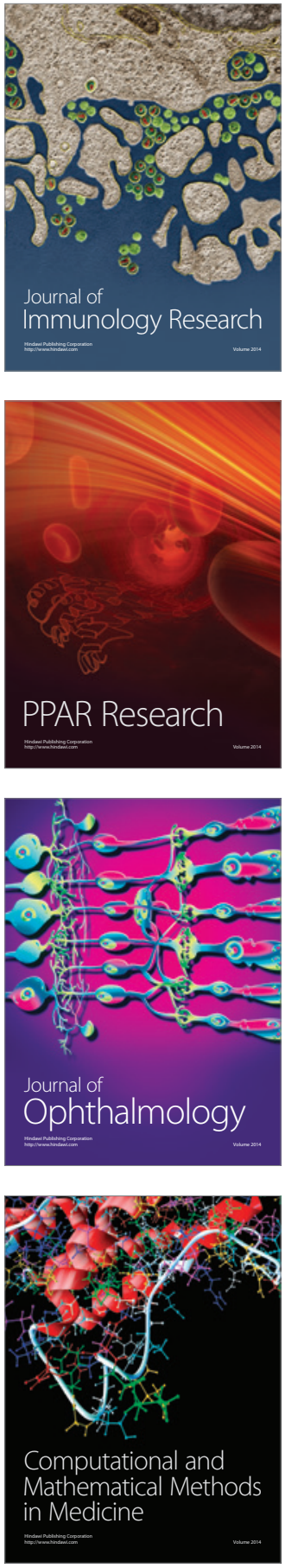

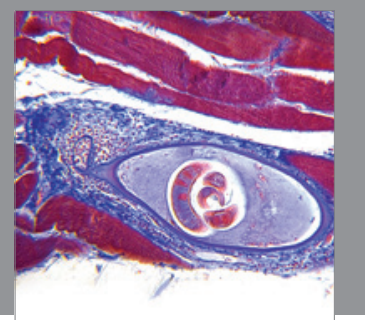

Gastroenterology

Research and Practice
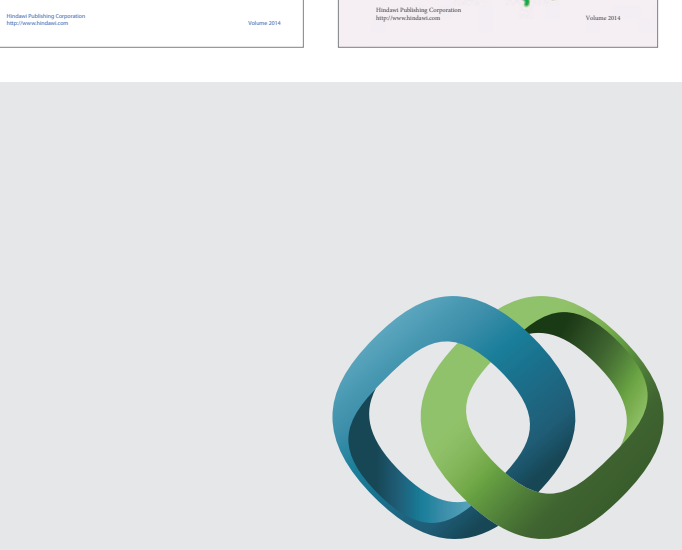

\section{Hindawi}

Submit your manuscripts at

http://www.hindawi.com
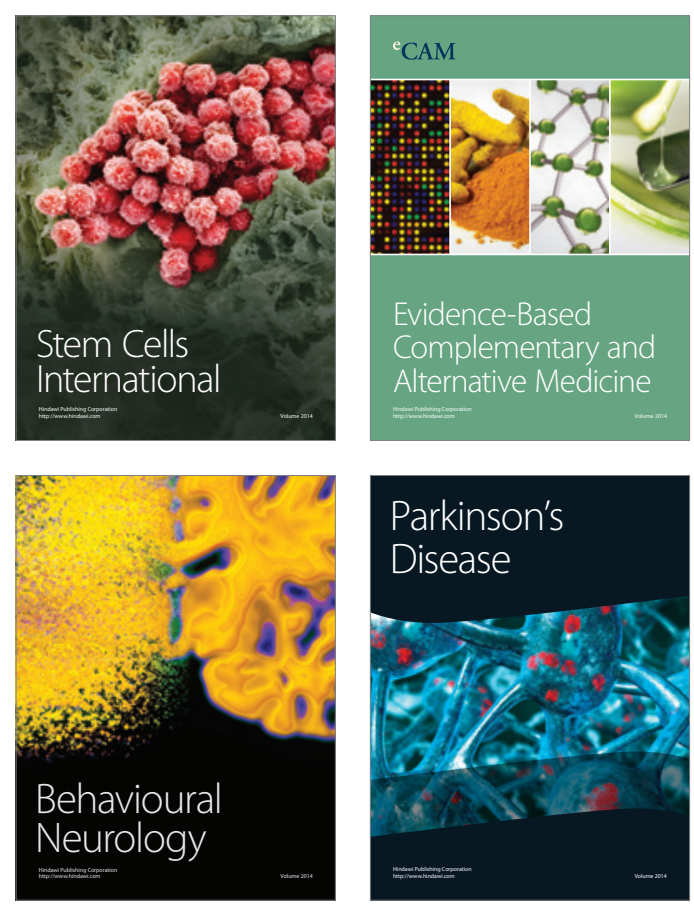

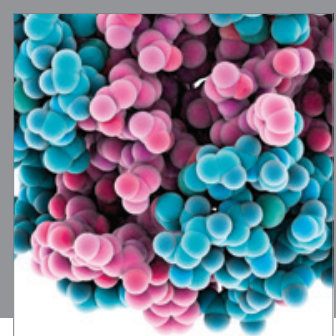

Journal of
Diabetes Research

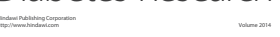

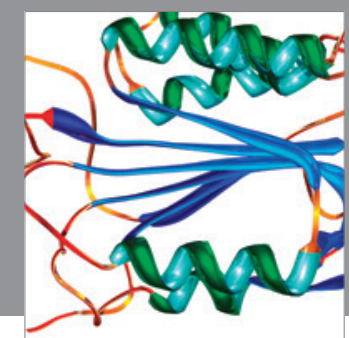

Disease Markers
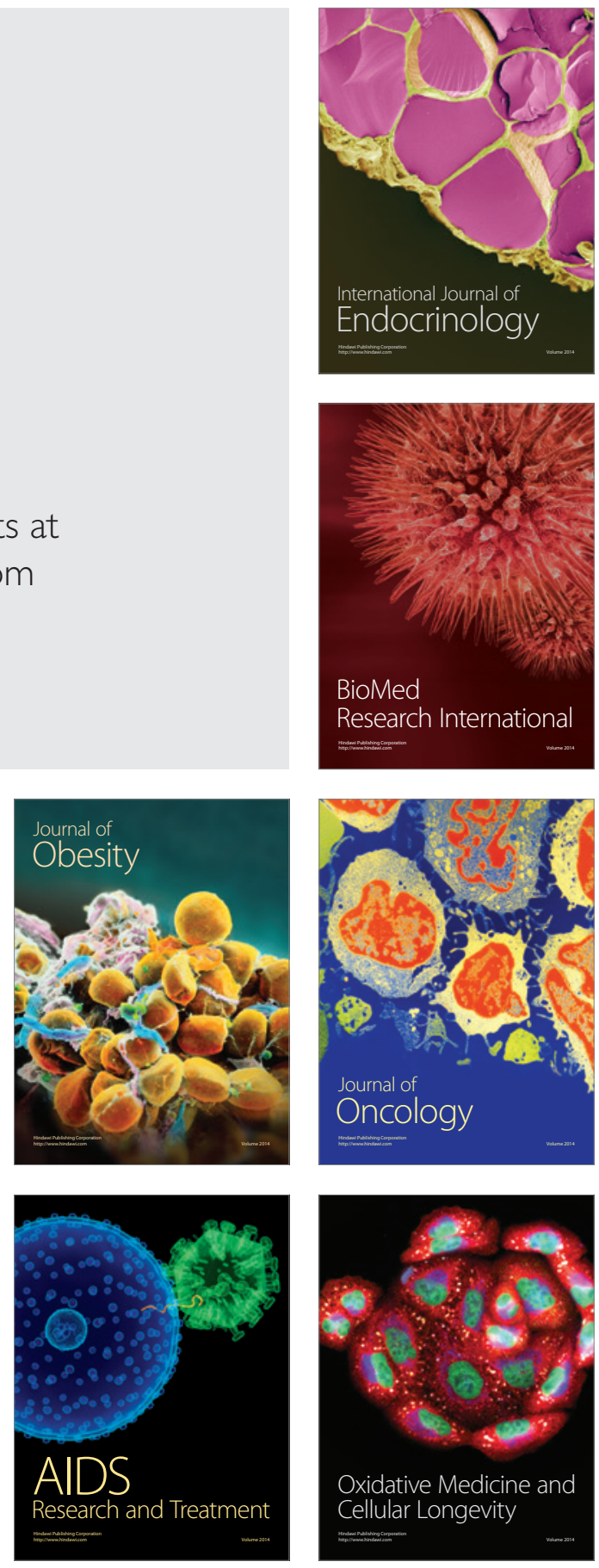